JOURNAL OF

SYMPLECTIC GEOMETRY

Volume 4, Number 2, 171-198, 2006

\title{
SYMBOLIC CALCULUS FOR TOEPLITZ OPERATORS WITH HALF-FORM
}

\author{
LAURENT Charles
}

\begin{abstract}
This paper is devoted to the use of half-form bundles in the symbolic calculus of Berezin-Toeplitz operators on Kähler manifolds. We state the Bohr-Sommerfeld conditions and relate them to the functional calculus of Toeplitz operators, a trace formula and the characteristic classes in deformation quantization. We also develop the symbolic calculus of Lagrangian sections, with the crucial estimates of the subprincipal terms.
\end{abstract}

In semi-classical analysis, we usually consider (pseudo) differential operators depending on a small parameter and acting on a $L^{2}$ space, the underlying classical limit being a cotangent space with its canonical symplectic structure. In this paper, we are interested in a similar theory where the classical phase space is a compact Kähler manifold endowed with a prequantum line bundle $L$. Here the quantum Hilbert space consists of the holomorphic sections of $L^{k}$. The small parameter is the inverse of $k$. The operators of interest are the Berezin-Toeplitz operators. This setting was mainly introduced by Kostant [16], Souriau [19] and Berezin [1] and the suitable microlocal techniques were developed by Boutet de Monvel and Guillemin [5]. Since then, many standard results for pseudo-differential operators have been adapted to this context, for instance, the Schnirelman theorem [22], the Gutzwiller trace formula [4] or the Bohr-Sommerfeld conditions [7]. The statements of these results are easily predictable as far as only the symplectic structure of the phase space is concerned because they are the same for the cotangent and Kähler spaces. But the semi-classical results for the pseudo-differential operators may involve also some invariants, like the subprincipal symbol or the Maslov index, which do not only depend on the symplectic structure and consequently are difficult to identify in the Kähler setting. Furthermore, these quantities generally appear as quantum 
corrections and are difficult to compute. Nevertheless in the papers $[\mathbf{6}]$ and [7], we carried out successfully some techniques to handle this. To formulate our result, we used the Riemannian metric of the Kähler structure instead of the vertical polarization of the cotangent bundle. Typically, we proved some Bohr-Sommerfeld conditions where the Maslov index is replaced with a curvature integral. Actually, we missed the right formulation which uses the half-form bundles. The main purpose of this paper and the sequel $[\mathbf{8}]$ is to develop this point of view. In this part, we focus on the Bohr-Sommerfeld conditions, whereas $[\mathbf{8}]$ is devoted to the dependence of the quantization on the complex structure.

Concretely we alter the usual setting by defining the quantum space as the space of holomorphic sections of $L^{k} \otimes L_{1} \otimes \delta \rightarrow M$. Here $L$ is the prequantum bundle as previously, $L_{1}$ is an auxiliary line bundle and $\delta$ is a half-form bundle, i.e. a square root of the canonical bundle of $M$. A priori artificial, this decomposition enlightens the semi-classical results, even in the usual case where $L_{1} \otimes \delta$ is the trivial bundle. Indeed in all our statements, one can clearly distinguish the contributions of $L_{1}$ and $\delta$. Typically, the contribution of $L_{1}$ in the semi-classical limit is the same as the one of $L^{k}$ (one can view $L^{k} \otimes L_{1}$ as a first-order deformation of $L^{k}$ ), whereas the contribution of the half-form bundle, if any, reflects the choice of the complex polarization. An important point is that there is a topological obstruction to the existence of half-form bundles. To avoid this problem, we consider globally the bundle $L^{k} \otimes K$ and write locally $K=L_{1} \otimes \delta$. The situation here is analogous to that in Riemannian geometry where we think any Clifford module, at least locally, as the spinor bundle twisted with an auxiliary bundle.

The first section is devoted to basic properties of Toeplitz operators and their symbolic calculus. In particular, an important subprincipal symbol is defined. We state the Bohr-Sommerfeld conditions in Section 2 and relate them to the symbolic calculus and trace formula by adapting an argument of Colin de Verdière [9]. Here, the formulation with half-forms permits to check easily the consistency of the results. The next sections contain the proof of the Bohr-Sommerfeld conditions. In Section 4, we introduce the Lagrangian sections, which are similar to the Lagrangian distributions, and develop their symbolic calculus. Bohr-Sommerfeld conditions follow immediately. A comparison with the usual setting is included, where a $\mathbb{Z}_{4}$-bundle plays a role analogous to the Maslov bundle. In Section 5 , the technical part of the paper, we provide the proof for the symbolic calculus of the Lagrangian sections. We follow essentially the method of [7] but avoid the complicated computations involving the derivatives of the Kähler metric. These simplifications rely on a version of the stationary phase lemma stated in the appendix of this paper. In view of this proof, we think that our result should generalize mutatis mutandis to the case where the symplectic manifold does not admit any integrable complex structure. 


\section{The setting}

1.1. Square root of line bundle. Let $M$ be a manifold and $F \rightarrow M$ be a complex line bundle. A square root $(\delta, \varphi)$ of $F$ is a line bundle $\delta \rightarrow M$ together with an isomorphism of line bundle $\varphi: \delta^{\otimes 2} \rightarrow F$. If $M$ is a complex manifold, a square root of its canonical bundle $\Lambda^{n, 0} T^{*} M$ is called a half-form bundle. Let us state basic properties of square roots.

If $F$ has a Hermitian structure and $(\delta, \varphi)$ is a square root of $F$, then $\delta$ has a unique Hermitian structure such that $\varphi$ is a isomorphism of Hermitian line bundle. In the same way, if $F$ is holomorphic or flat, $\delta$ inherits the same structure. If $D^{F}$ is a first-order differential operator acting on sections of $F \rightarrow M$, then there exists a unique first-order differential operator $D^{\delta}$ acting on section of $\delta$ such that

$$
D^{F} \varphi(s \otimes s)=2 \varphi\left(s \otimes D^{\delta} s\right), \quad \forall s \in C^{\infty}(M, \delta) .
$$

A line bundle admits a square root if and only if its Chern class is divisible by 2 in $H^{2}(M, \mathbb{Z})$. Two square roots $(\delta, \varphi)$ and $\left(\delta^{\prime}, \varphi^{\prime}\right)$ of $F$ are equivalent if there exists an isomorphism $\Psi: \delta \rightarrow \delta^{\prime}$ such that $\varphi^{\prime} \circ \Psi^{2}=\varphi$.

Proposition 1.1. Assume that $F$ admits a square root. Then the set of equivalence classes of square roots of $F$ is a principal homogeneous space for the first group of cohomology of $M$ with coefficient in $\mathbb{Z}_{2}$.

Proof. First, if $(\delta, \varphi)$ is a square root of the trivial line bundle $1_{M}=M \times \mathbb{C}$, then $\delta$ inherits a flat structure from $1_{M}$ with structure group $\mathbb{Z}_{2}$. Furthermore, this flat structure determines $\varphi$. It is easily proved that this induces an isomorphism between the set of equivalence classes of square roots of $1_{M}$ and the set of equivalences of flat line bundles with structure group $\mathbb{Z}_{2}$. The latter is isomorphic to $H^{1}\left(M, \mathbb{Z}_{2}\right)$. Now, observe that the tensor product of a square root of $L$ with a square root of $1_{M}$ is a square root of $L$. This defines an action of $H^{1}\left(M, \mathbb{Z}_{2}\right)$ on $\mathcal{E}_{L}$, which is easily shown to be free and transitive.

1.2. Quantum spaces. Let $M$ be a connected compact Kähler manifold of complex dimension $n$. Denote by $\omega \in \Omega^{2}(M, \mathbb{R})$ the fundamental form of $M$. Assume $M$ is endowed with a prequantization bundle

$$
L \longrightarrow M,
$$

that is a Hermitian line bundle with a connection $\nabla^{L}$ of curvature $\frac{1}{i} \omega$. Since $\omega$ is a $(1,1)$-form, $L$ has a natural holomorphic structure defined in such a way that the (local) holomorphic sections satisfy the Cauchy-Riemann equations: $\nabla_{\bar{Z}} s=0$ for every holomorphic vector field $Z$ of $M$.

Let $K \rightarrow M$ be a Hermitian holomorphic line bundle. For every positive integer $k$, define the quantum space $\mathcal{H}_{k}$ :

$$
\mathcal{H}_{k}=\left\{\text { holomorphic section of } L^{k} \otimes K\right\} \text {. }
$$


Assume that $M$ carries a half-form bundle $(\delta, \varphi) . \quad \delta \rightarrow M$ inherits a Hermitian scalar product and a holomorphic structure from $\Lambda^{n, 0} T^{*} M$. Introduce the Hermitian holomorphic line bundle $L_{1}$ such that

$$
K=L_{1} \otimes \delta
$$

and let $\frac{1}{i} \omega_{1}$ be the curvature of the Chern connection of $L_{1}$.

Since $M$ is compact, $\mathcal{H}_{k}$ is finite dimensional, and it follows from the Riemann-Roch-Hirzebruch theorem and Kodaira vanishing theorem that

$$
\operatorname{dim} \mathcal{H}_{k}=\left(\frac{k}{2 \pi}\right)^{n} \int_{M} \frac{\left(\omega+k^{-1} \omega_{1}\right)^{\wedge n}}{n !}+O\left(k^{n-2}\right) .
$$

To interpret this formula, we consider $L^{k} \otimes L_{1}$ and $\omega+\hbar \omega_{1}$ as deformations of $L^{k}$ and $\omega$ which give the first quantum corrections in the semi-classical limit. Indeed, the leading term

$$
\left(\frac{k}{2 \pi}\right)^{n} \int \frac{\omega^{\wedge n}}{n !}
$$

gives the second-order correction when we replace $\omega$ with $\omega+k^{-1} \omega_{1}$. Furthermore, in the case $M$ does not carry any half-form bundle, equation (1.1) is still valid if we define $\omega_{1}$ by

$$
\omega_{1}:=\omega_{K}-\frac{\omega_{c}}{2},
$$

where $\frac{1}{i} \omega_{K}$ and $\frac{1}{i} \omega_{c}$ are the curvatures of the Chern connections of $K$ and $\Lambda^{n, 0} T^{*} M$.

1.3. Toeplitz operators. Let $\Pi_{k}$ be the orthogonal projector of $L^{2}\left(M, L^{k}\right.$ $\otimes K$ ) onto $\mathcal{H}_{k}$, where the scalar product of two sections of $L^{k} \otimes K$ is defined from the Hermitian structures of $L$ and $K$ and the Liouville form $\mu_{M}$.

A Toeplitz operator is any sequence $\left(T_{k}: \mathcal{H}_{k} \rightarrow \mathcal{H}_{k}\right)$ of operators of the form

$$
T_{k}=\Pi_{k} f(\cdot, k)+R_{k},
$$

where $f(\cdot, k)$ is a sequence of $C^{\infty}(M)$ with an asymptotic expansion $f_{0}+$ $k^{-1} f_{1}+\cdots$ for the $C^{\infty}$ topology and the norm of $R_{k}$ is $O\left(k^{-\infty}\right)$.

The set $\mathcal{T}$ of Toeplitz operators is a semi-classical algebra associated to $(M, \omega)$ in the following sense.

Theorem 1.2. $\mathcal{T}$ is closed under the formation of product. So it is a star algebra, the identity is $\left(\Pi_{k}\right)$. The symbol map

$$
\sigma_{\text {cont }}: \mathcal{T} \longrightarrow C^{\infty}(M)[[\hbar]],
$$

sending $T_{k}$ into the formal series $f_{0}+\hbar f_{1}+\cdots$, where the functions $f_{i}$ are the coefficients of the asymptotic expansion of the multiplicator $f(\cdot, k)$, 
is well defined. It is onto and its kernel is the ideal consisting of $O\left(k^{-\infty}\right)$ Toeplitz operators. More precisely, for any integer $\ell$,

$$
\left\|T_{k}\right\|=O\left(k^{-\ell}\right) \text { if and only if } \sigma_{\text {cont }}\left(T_{k}\right)=O\left(\hbar^{\ell}\right) .
$$

Furthermore, the induced product $*_{\text {cont }}$ on $C^{\infty}(M)[[\hbar]]$ is a star-product.

Following the terminology of Berezin in [1] , we call $\sigma_{\text {cont }}$ the contravariant symbol map. This result is essentially a consequence of the works of Boutet de Monvel and Guillemin [5] (cf. also [3, 6, 12]). Let us recall that equivalence classes of star-products on $(M, \omega)$ are parametrized by elements in

$$
\frac{1}{i \hbar}[\omega]+H^{2}(M, \mathbb{C})[[\hbar]]
$$

called Fedosov characteristic classes. The following theorem was proved by Karabegov and Schlichenmaier in [14] and [15], in the case $K$ is the trivial line bundle.

Theorem 1.3. The Fedosov class of the star-product $*_{\operatorname{cont}}$ is $\frac{1}{i \hbar}\left([\omega]+\hbar\left[\omega_{1}\right]\right)$.

Again, it is interesting to note the appearance of $\omega+\hbar \omega_{1}$. We do not need this result but some related facts. Let us define the normalized symbol of a Toeplitz operator by

$$
\sigma_{\text {norm }}\left(T_{k}\right):=\left(\operatorname{Id}+\frac{\hbar}{2} \Delta\right) \sigma_{\text {cont }}\left(T_{k}\right),
$$

where $\Delta$ is the holomorphic Laplacian acting on $C^{\infty}(M)$. Actually we are only interested in the leading and second-order terms of $\sigma_{\text {norm }}\left(T_{k}\right)$, and modifying the definition of $\sigma_{\text {norm }}\left(T_{k}\right)$ by a $O\left(\hbar^{2}\right)$ term would not change the statements of our results. To compare with our previous article [7], the Weyl symbol that we introduced when $K$ is the trivial line bundle is equal to the normalized symbol modulo $O\left(\hbar^{2}\right)$.

The map $\sigma_{\text {norm }}: \mathcal{T} \rightarrow C^{\infty}(M)[[\hbar]]$ satisfies the same properties as $\sigma_{\text {cont }}$ stated in Theorem 1.2. Denote by $*_{\text {norm }}$ the associated star product.

Theorem 1.4. Let $f$ and $g$ belong to $C^{\infty}(M)[[\hbar]]$. Then,

$$
f *_{\text {norm }} g=f \cdot g+\frac{\hbar}{2 i}\langle\pi, d f \wedge d g\rangle+O\left(\hbar^{2}\right)
$$

and

$$
i \hbar^{-1}\left(f *_{\text {norm }} g-g *_{\text {norm }} f\right)=\left\langle\pi+\hbar \pi_{1}, d f \wedge d g\right\rangle+O\left(\hbar^{2}\right) .
$$

where $\pi$ is the Poisson bivector and $\pi_{1}$ is the bivector such that $\left\langle\pi_{1}, d f \wedge d g\right\rangle+$ $\left\langle X_{f} \wedge X_{g}, \omega_{1}\right\rangle=0$ for every $f, g \in C^{\infty}(M)$.

So $*_{\text {norm }}$ is a normalized star-product, in the sense that the second-order term in the first formula is anti-symmetric, which explains our terminology. 
Observe that $\pi+\hbar \pi_{1}$ is the Poisson bivector associated to $\omega+\hbar \omega_{1}$ in the sense that

$$
\left\langle\pi+\hbar \pi_{1}, d f \wedge d g\right\rangle=\left\langle\left(X_{f}+\hbar X_{f}^{1}\right) \wedge\left(X_{g}+\hbar X_{g}^{1}\right), \omega+\hbar \omega_{1}\right\rangle+O\left(\hbar^{2}\right),
$$

where $X_{f}+\hbar X_{f}^{1}+O\left(\hbar^{2}\right)$ is the Hamiltonian vector field of $f$ with respect to $\omega+\hbar \omega_{1}$, that is,

$$
d f+\left\langle\omega+\hbar \omega_{1}, X_{f}+\hbar X_{f}^{1}\right\rangle=O\left(\hbar^{2}\right),
$$

and the same holds for $g$ and $X_{g}+\hbar X_{g}^{1}$. Thus, Theorem 1.3 implies that there exists a star product equivalent to $*_{\text {cont }}$ and satisfying the formulas of Theorem 1.4. As a partial converse, Theorem 1.4 implies that the Fedosov class of $*_{\text {cont }}$ is $\frac{1}{i \hbar}\left([\omega]+\hbar\left[\omega_{1}\right]+O\left(\hbar^{2}\right)\right)$. Furthermore, the equivalence $\operatorname{Id}+\frac{\hbar}{2} \Delta$ is specified.

We can prove Theorem 1.4 using the methods of $[\mathbf{6}, \mathbf{1 5}]$. But this leads to complicated computations. We will present in [8] a more conceptual proof. We stated this result because we can deduce from it a part of the Bohr-Sommerfeld conditions (cf. Sections 2.2, 2.3 and 2.4).

1.4. Relation with geometric quantization. Our definition of the normalized symbol agrees in some sense with the usual procedure to quantize observables in geometric quantization. Assume that the Hamiltonian flow of $f \in C^{\infty}(M)$ preserves the complex structure of $M$. Assume also that $K=L_{1} \otimes \delta$, where $(\delta, \varphi)$ is a half-form bundle. Then the following operator is well defined:

$$
\mathcal{Q}(f):=f+\frac{1}{i k}\left(\nabla_{X_{f}}^{L^{k} \otimes L_{1}} \otimes \operatorname{Id}+\operatorname{Id} \otimes \mathcal{L}_{X_{f}}\right): \mathcal{H}_{k} \longrightarrow \mathcal{H}_{k} .
$$

Here $\mathcal{L}_{X}$ acts on sections of $\delta$ by $\varphi\left(\left(\mathcal{L}_{X} s\right) \otimes s\right)=\frac{1}{2} \mathcal{L}_{X} \varphi\left(s^{\otimes 2}\right)$ or equivalently as a Lie derivative where the pull-back of sections of $\delta$ by a complex diffeomorphism $\zeta$ is defined in such a way that $\varphi\left(\left(\zeta^{*} s\right)^{\otimes 2}\right)=\zeta^{*} \varphi\left(s^{\otimes 2}\right)$.

The definition (1.2) is natural for the following reason. Denote by $\Phi_{t}$ the Hamiltonian flow of $X_{f}$. Let $\tilde{\Phi}_{t}$ be the lift of $\Phi_{t}$ to $L^{k} \otimes L_{1} \otimes \delta$ defined by the tensor product of the parallel transport along the trajectories of $X_{f}$ in $L^{k} \otimes L_{1}$ and by the pull-back in $\delta$. Then the solution of the Schrödinger equation

$$
\frac{1}{i k} \frac{d}{d t} \Psi(\cdot, t)+\mathcal{Q}(f) \Psi(\cdot, t)=0,
$$

with initial condition $\Psi \in \mathcal{H}_{k}$ is given by

$$
\Psi(x, t)=\mathrm{e}^{(k / i) \int_{0}^{t} f\left(\Phi_{s-t}(x)\right) d s} \tilde{\Phi}_{t}\left(\Psi\left(\Phi_{-t}(x)\right)\right) .
$$

The important point for us is that $\mathcal{Q}(f)$ is a Toeplitz operators whose normalized symbol is $f$ modulo $O\left(\hbar^{2}\right)$. We will prove a more general result for every smooth function $f$, which simplifies some further proofs. 
If $f$ is an arbitrary smooth function, formula (1.2) does not make sense because the Lie derivative with respect to $X_{f}$ does not necessarily preserve $\Omega^{n, 0}(M)$. So we define for any vector field $X$ the operator $D_{X}$,

$$
D_{X} \alpha=p\left(\mathcal{L}_{X} \alpha\right), \quad \alpha \in \Omega^{n, 0}(M),
$$

where $p$ is the projection from $\Lambda^{n} T^{*} M \otimes \mathbb{C}$ onto $\Lambda^{n, 0} T^{*} M$ with kernel the sum

$$
\Lambda^{n-1,1} T^{*} M \oplus \Lambda^{n-2,2} T^{*} M \oplus \cdots \oplus \Lambda^{0, n} T^{*} M .
$$

Next, we let $D_{X}$ act on the sections of $\delta$ as the first-order differential operator such that $2 \varphi\left(s \otimes D_{X} s\right)=D_{X} \varphi\left(s^{2}\right)$.

Theorem 1.5. For any $f \in C^{\infty}(M)$, the operator

$$
\mathcal{Q}(f):=\Pi_{k}\left(f+\frac{1}{i k}\left(\nabla_{X_{f}}^{L^{k} \otimes L_{1}} \otimes \mathrm{Id}+\mathrm{Id} \otimes D_{X_{f}}\right)\right): \mathcal{H}_{k} \longrightarrow \mathcal{H}_{k}
$$

is a Toeplitz operator with principal symbol $f$ and vanishing subprincipal symbol.

This theorem is a consequence of the following lemma and an argument of Tuynman $[\mathbf{2 0}]$.

Lemma 1.6. Let $s$ be a half-form. Then,

$$
D_{X_{f}} s=\nabla_{X_{f}}^{\delta} s+\frac{i}{2}(\Delta f) s,
$$

where $\nabla^{\delta}$ is the Chern connection of $\delta$, and $\Delta$ is the holomorphic Laplacian of $M$.

It follows that

$$
\nabla_{X_{f}}^{L^{k} \otimes L_{1}} \otimes \mathrm{Id}+\mathrm{Id} \otimes D_{X_{f}}=\nabla_{X_{f}}^{L^{k} \otimes L_{1} \otimes \delta}+\frac{i}{2}(\Delta f)=\nabla_{X_{f}}^{L^{k} \otimes K}+\frac{i}{2}(\Delta f) .
$$

Now we have for every $\Psi \in \mathcal{H}_{k}$,

$$
\Pi_{k}\left(\nabla_{X_{f}}^{L^{k} \otimes K} \Psi\right)=\frac{1}{i} \Pi_{k}(\Delta f \cdot \Psi),
$$

cf. $[\mathbf{3}, \mathbf{2 0}]$ for a proof. Hence

$$
\mathcal{Q}(f) \Psi=\Pi_{k}\left(\left(f-\frac{1}{2 k} \Delta f\right) \Psi\right)
$$

which proves Theorem 1.5.

The last expression in (1.3) shows that the definition of $\mathcal{Q}(f)$ is independent of the choice of the half-form bundle and generalizes in the cases where no such bundle exists. 
Proof of lemma 1.6. It suffices to prove that for every $\alpha \in \Omega^{n, 0}(M)$, we have

$$
D_{X_{f}} \alpha=\nabla_{X_{f}} \alpha+i(\Delta f) \alpha .
$$

Introduce normal complex coordinates $z^{1}, \ldots, z^{n}$ centered at $x_{0}$. So, if $\alpha=$ $d z^{1} \wedge \ldots \wedge d z^{n}$, then $\nabla \alpha=0$ at $x_{0}$. Let us write $\omega=i G_{j, k} d z^{j} \wedge d \bar{z}^{k}$. Then,

$$
X_{f}=-i G^{j, k}\left(\partial_{z^{j}} f\right) \partial_{\bar{z}^{k}}+i G^{j, k}\left(\partial_{\bar{z}^{k}} f\right) \partial_{z^{j}} \text {. }
$$

Using that the first derivatives of $G^{j, k}$ vanish at $x_{0}$, we obtain easily

$$
D_{X_{f}} \alpha=i G^{j, k}\left(\partial_{z^{j}} \partial_{\bar{z}^{k}} f\right)=i \Delta f .
$$

The result follows.

\section{Bohr-Sommerfeld conditions}

2.1. The result. Assume that $M$ is two-dimensional. When possible, the generalizations to any dimension will be precised. Let $(\delta, \varphi)$ be a half-form bundle and let us write $K=L_{1} \otimes \delta$ as previously. Consider a self-adjoint Toeplitz operator $\left(T_{k}\right)$. Its normalized symbol

$$
f_{0}+\hbar f_{1}+\cdots
$$

is real-valued. Bohr-Sommerfeld conditions give the spectrum of $T_{k}$ on every open interval $I$ of regular values of $f_{0}$ in the semi-classical limit. To simplify the statements, assume that $f_{1}$ vanishes.

Let $\Gamma^{1}, \ldots, \Gamma^{m}$ be the components of $f_{0}^{-1}(I)$. For every $i \in\{1, \ldots, m\}$, the map

$$
f_{0}: \Gamma^{i} \longrightarrow I
$$

is a trivial fibration with fiber diffeomorphic to $S^{1}$. For every $\lambda \in I$, fix an orientation on the fiber $\Gamma_{\lambda}^{i}=f_{0}^{-1}(\lambda) \cap \Gamma^{i}$ depending continuously on $\lambda$.

Let $a^{i} \in C^{\infty}(I)$ be the principal action, defined in such a way that the parallel transport in $L$ along $\Gamma_{\lambda}^{i}$ is the multiplication by $\exp \left(i a^{i}(\lambda)\right)$. Using $L_{1}$ instead of $L$, define in the same way the subprincipal action $a_{1}^{i} \in C^{\infty}(I)$.

Let us define an index $\epsilon^{i}$ from the half-form bundle $(\delta, \varphi)$. Observe that the restriction $\delta_{i, \lambda}$ of $\delta$ to $\Gamma_{\lambda}^{i}$ is a square root of $T^{*} \Gamma_{\lambda}^{i} \otimes \mathbb{C}$. Indeed, let us denote by $\iota$ the embedding $\Gamma_{\lambda}^{i} \rightarrow M$. Then the map

$$
\varphi_{i, \lambda}: \delta_{i, \lambda}^{2} \longrightarrow T^{*} \Gamma_{\lambda}^{i} \otimes \mathbb{C}, \quad u \longrightarrow \iota^{*} \varphi(u)
$$

is an isomorphism of line bundle. The set

$$
\left\{u \in \delta_{i, \lambda} ; \varphi_{i, \lambda}\left(u^{\otimes 2}\right)>0\right\}
$$

has one or two connected components. In the first case, we set $\epsilon_{\lambda}^{i}=1$ and in the second case $\epsilon_{\lambda}^{i}=0$. Observe that $\epsilon_{\lambda}^{i}$ does not depend on $\lambda$.

The Bohr-Sommerfeld conditions are

$$
a^{i}(\lambda)+k^{-1}\left(a_{1}^{i}(\lambda)+\epsilon^{i} \pi\right) \in \frac{2 \pi}{k} \mathbb{Z} .
$$


Denote by $\Sigma^{i}(k)$ the set of $\lambda \in I$ satisfying (2.1). When $k$ is sufficiently large, $\Sigma^{i}(k)$ is a finite set containing

$$
\frac{k}{2 \pi} \operatorname{Vol}\left(\Gamma^{i}\right)+O\left(k^{-1}\right)
$$

points. Let $\Sigma(k)$ be the union of the $\Sigma^{i}(k)$. Define the multiplicity of $\lambda \in \Sigma(k)$ as the number of $\Sigma^{i}(k)$ which contains $\lambda$. The points of $\Sigma(k)$ approximate the eigenvalues of $T_{k}$ in the following sense.

Theorem 2.1. Let $\lambda_{-}(k)$ and $\lambda_{+}(k)$ be two sequences of $I$ such that

$$
d\left(\lambda_{-}(k), \Sigma(k)\right) \geqslant C k^{-1}, \quad d\left(\lambda_{+}(k), \Sigma(k)\right) \geqslant C k^{-1}
$$

for some positive $C$. Assume furthermore that there exists $\lambda_{-}, \lambda_{+} \in I$ such that

$$
\lambda_{-} \leqslant \lambda_{-}(k) \leqslant \lambda_{+}(k) \leqslant \lambda_{+} .
$$

Denote by $\lambda_{1}(k) \leqslant \lambda_{2}(k) \leqslant \cdots \leqslant \lambda_{N(k)}(k)$ (resp. $\lambda_{1}^{\prime}(k) \leqslant \lambda_{2}^{\prime}(k) \leqslant \cdots \leqslant$ $\lambda_{N^{\prime}(k)}^{\prime}(k)$ ) the eigenvalues of $\left(T_{k}\right)$ (resp. points of $\Sigma(k)$ ) contained in $\left(\lambda_{-}(k), \lambda_{+}(k)\right)$ and counted with multiplicities. Then, when $k$ is sufficiently large, $N(k)=N^{\prime}(k)$. Furthermore,

$$
\lambda_{j}(k)=\lambda_{j}^{\prime}(k)+O\left(k^{-2}\right)
$$

uniformly with respect to $j$.

This result can be generalized to any dimension with a suitable and more restrictive assumption to describe the joint spectrum of $n$ commuting Toeplitz operators (cf. Section 3.2, equation (3.3)).

The interest of condition (2.2) is to avoid any ambiguity in the counting of eigenvalues near the endpoints of $\left(\lambda_{-}(k), \lambda_{+}(k)\right)$. It is not restrictive. Indeed if $\lambda_{-}(k), \lambda_{+}(k)$ are arbitrary sequences satisfying (2.3), then by modifying them by suitably chosen $O\left(k^{-1}\right)$ sequences, we obtain sequences satisfying both estimates (2.2) and (2.3).

Since the definition of the Toeplitz operators and of their normalized symbol only depend on $K$ and not on the choice of the half-form bundle, it is likely that the same holds for the Bohr-Sommerfeld conditions. This is easily checked using that any other half-form bundle is of the form $\delta^{\prime}=\delta \otimes F$, where $F$ is a flat Hermitian line bundle with holonomy in $\mathbb{Z}_{2}$. So $L_{1}^{\prime}=L_{1} \otimes F^{-1}$, and straightforward computations show that the functions $a_{1}^{i}+\epsilon^{i}$ do not depend on the choice of $\delta$.

To compare with our previous results in [7], when $K$ is the trivial bundle, we defined the function $a_{1}^{i}+\epsilon^{i}$ as the integral of the geodesic curvature of $\Gamma_{\lambda}^{i}$.

We can also approximate the eigenvalues up to a $O\left(k^{-\infty}\right)$ error. More precisely, there exist sequences $\left(S^{i}(\cdot, k)\right)_{k}$ of $C^{\infty}(I)$ such that the BohrSommerfeld conditions

$$
S^{i}(\cdot, k) \in \frac{2 \pi}{k} \mathbb{Z}
$$


instead of (2.1) lead to the same result with a $O\left(k^{-\infty}\right)$ error in (2.4). Furthermore, the sequences $S^{i}(\cdot, k)$ admit asymptotic expansions of the form

$$
a^{i}+k^{-1}\left(a_{1}^{i}+\epsilon^{i} \pi\right)+k^{-2} S_{2}^{i}+k^{-3} S_{3}^{i}+\cdots .
$$

Applying an argument of Colin de Verdière [9], we can prove that the derivatives of the first two coefficients and of the $S_{j}^{i}$ only depend on the star-product $*_{\text {norm }}$ and the normalized symbol of $T_{k}$. In particular, we will deduce from Theorem 1.4 the following

Proposition 2.2. Let $\tilde{S}_{0} \in C^{\infty}(I)$ be increasing and $\tilde{S}_{1} \in C^{\infty}(I)$. Assume that $f_{0}^{-1}(I)$ is connected and that the conclusions of Theorem 2.1 hold with

$$
\tilde{\Sigma}(k)=\left\{\lambda \in I ; \tilde{S}_{0}(\lambda)+k^{-1} \tilde{S}_{1}(\lambda) \in \frac{2 \pi}{k} \mathbb{Z}\right\}
$$

instead of $\Sigma(k)$. Then, one has

$$
\tilde{S}_{0}(\lambda)-\tilde{S}_{0}\left(\lambda^{\prime}\right)=\int_{D} \omega \quad \text { and } \quad \tilde{S}_{1}(\lambda)-\tilde{S}_{1}\left(\lambda^{\prime}\right)=\int_{D} \omega_{1}
$$

if $\lambda, \lambda^{\prime} \in I$ are such that $\lambda^{\prime} \leqslant \lambda$ and $D=\Gamma \cap f_{0}^{-1}\left(\left(\lambda, \lambda^{\prime}\right)\right)$.

Of course, since the curvature of $L$ and $L_{1}$ are $\frac{1}{i} \omega$ and $\frac{1}{i} \omega_{1}$, respectively, the previous proposition follows directly from Theorem 2.1. The interesting point is that we can prove it from the formulas for the star-product $*_{\text {norm }}$ of Theorem 1.4. Note that only the derivatives of $S_{0}^{i}$ and $S_{1}^{i}$ are determined by the star-product. Indeed, if we twist $L$ or $L_{1}$ by a flat Hermitian line bundle, the actions of the non-contractible loops may change although the star-product remains the same.

The proof of (2.5) is in Section 2.4, and the next two sections contain preliminary results on traces and functional calculus of Toeplitz operators. The proof of Theorem 2.1 is postponed to Section 3.2.

2.2. Traces. In this section and the next one, we do not necessarily assume that $M$ is two-dimensional. It is a known result that the trace of a Toeplitz operator $T_{k}$ with normalized symbol $f_{0}+\hbar f_{1}+\cdots$ admits an asymptotic expansion of the following form:

$\operatorname{Tr}\left(T_{k}\right)=\left(\frac{k}{2 \pi}\right)^{n} \int_{M}\left(f_{0}+k^{-1} f_{1}+\cdots\right)\left(1+k^{-1} d_{1}+k^{-2} d_{2}+\cdots\right) \mu_{M}+O\left(k^{-\infty}\right)$,

where $d_{1}, d_{2}, d_{3}, \ldots$ are functions of $C^{\infty}(M)$ which do not depend of $T_{k}$ (cf., for instance, $[6])$.

These functions may be computed in terms of the Kähler metric by using the methods of $[\mathbf{6}]$, but it is more convenient to relate them to the starproduct $*_{\text {norm}}$. To do this, observe that the $\mathbb{C}[[\hbar]]$-linear map

$$
\left.\operatorname{tr}: C^{\infty}(M)[[\hbar]] \longrightarrow \mathbb{C}\left[\hbar^{-1}, \hbar\right]\right], \quad f(\hbar) \longrightarrow(2 \pi \hbar)^{-n} \int_{M} f(\hbar) d(\hbar) \mu_{M},
$$


where $d(\hbar)=1+\hbar d_{1}+\hbar^{2} d_{2}+\cdots$ is a trace for the star-product $*_{\text {norm }}$, in the sense that it satisfies

$$
\operatorname{tr}\left(f *_{\text {norm }} g\right)=\operatorname{tr}\left(g *_{\text {norm }} f\right) .
$$

Following Fedosov [11] or Nest and Tsygan [18], such a trace is unique up to multiplication by an element of $\left.\mathbb{C}\left[\hbar^{-1}, \hbar\right]\right]$ and there exists a canonical one determined by the following normalization condition: for every local equivalence $\Phi$ between $*_{\text {norm }}$ and the Weyl star-product, we have

$$
\operatorname{tr}(f)=(2 \pi \hbar)^{-n} \int_{M} \Phi(f) \mu_{M}, \quad \text { where } \mu_{M}=\frac{\omega^{n}}{n !} .
$$

We claim that the trace defined in (2.6) is the canonical trace. This follows from the fact that the quantization by Toeplitz operators is microlocally equivalent to the usual Weyl quantization.

So the functions $d_{i}$ are determined by $*_{\text {norm. }}$. In particular, it follows from Theorem 1.4 that

$$
\operatorname{tr}(f)=(2 \pi \hbar)^{-n} \int_{M} \frac{f\left(\omega+\hbar \omega_{1}\right)^{\wedge n}}{n !}+O\left(\hbar^{-n+2}\right)
$$

Proof of formula (2.8). Consider an equivalence of star-product of the form

$$
\Phi=\mathrm{Id}+\hbar X+O\left(\hbar^{2}\right),
$$

where $X$ is a vector field. It is easily checked that the star-product

$$
f *^{\prime} g=\Phi\left(\Phi^{-1}(f) * \text { norm } \Phi^{-1}(g)\right)
$$

is normalized and satisfies

$$
i \hbar^{-1}\left(f *^{\prime} g-g *^{\prime} f\right)=\left\langle\pi+\hbar\left(\pi_{1}+X \cdot \pi\right), d f \wedge d g\right\rangle+O\left(\hbar^{2}\right) .
$$

Locally, on can choose $X$ in such a way that $\pi_{1}+X \cdot \pi=0$. Indeed, this equation is equivalent to $\omega_{1}+d \alpha=0$, where $\alpha$ is the 1 -form such that $\omega(X, \cdot)=\alpha$.

Next step is to introduce local Darboux coordinates, which define a Weyl star-product $*_{\text {Weyl }}$. And modifying $\Phi$ by a $O\left(\hbar^{2}\right)$ term, one has $*_{\text {Weyl }}=*^{\prime}$ $[\mathbf{2}]$. Then it follows from (2.7) that

$$
\operatorname{tr}(f)=(2 \pi \hbar)^{-n} \int_{M} f\left(\mu_{M}-\hbar\left(X \mu_{M}\right)+O\left(\hbar^{2}\right)\right) .
$$

By definition of $\pi_{1}$ in Theorem 1.4, one has $\left\langle\pi_{1}, \omega\right\rangle+\left\langle\pi, \omega_{1}\right\rangle=0$. Since $X \cdot\langle\pi, \omega\rangle=0$, we obtain

$$
\langle\pi, X \omega\rangle=-\langle X \pi, \omega\rangle=\left\langle\pi_{1}, \omega\right\rangle=-\left\langle\omega_{1}, \pi\right\rangle .
$$

Then, a straightforward computation leads to

$$
\left\langle\pi^{\wedge n}, \mu_{M}-\hbar X \mu_{M}\right\rangle=\left\langle\pi^{\wedge n}, \frac{\left(\omega+\hbar \omega_{1}\right)^{\wedge n}}{n !}\right\rangle+O\left(\hbar^{2}\right),
$$

which proves the result. 
As a consequence of (2.8), we obtain the estimate (1.1) of the dimension of $\mathcal{H}_{k}$, since this dimension is the trace of the projector $\Pi_{k}$, whose normalized symbol is 1 . Actually the index theorems of deformation quantization proved in $[\mathbf{1 1}, \mathbf{1 8}]$ yield the asymptotic expansion of $\operatorname{Tr}\left(\Pi_{k}\right)$ modulo $O\left(k^{-\infty}\right)$ in terms of the Fedosov class of $*_{\text {norm. }}$. Since this trace is an integer, the $O\left(k^{-\infty}\right)$ error vanishes when $k$ is sufficiently large. In this way, we can deduce Riemann-Roch-Hirzebruch theorem from Theorem 1.3.

2.3. Functional calculus. Let $T_{k}$ be a self-adjoint Toeplitz operator with normalized symbol

$$
f=f_{0}+\hbar f_{1}+\cdots
$$

and let $g$ be a function of $C^{\infty}(\mathbb{R}, \mathbb{C})$. Then, it is known that $g\left(T_{k}\right)$ is a Toeplitz operator (cf., for instance, $[\mathbf{6}]$ ). Furthermore, the normalized symbol of $g\left(T_{k}\right)$ is given by the following non-commutative Taylor formula:

$$
g^{*_{\mathrm{norm}}}(f)(x)=\left.\sum \frac{1}{\ell !} g^{(\ell)}\left(f_{0}(x)\right)\left(f(y)-f_{0}(x)\right)^{*_{\mathrm{norm}} \ell}\right|_{y=x},
$$

where $g^{(\ell)}$ is the $\ell$ th derivative of $g$ and $h^{* \text { norm } \ell}=h *_{\text {norm }} \cdots *_{\text {norm }} h$ repeated $\ell$ times. In particular, an easy computation from Theorem 1.4 leads to

$$
g^{*_{\text {norm }}}(f)=g\left(f_{0}\right)+\hbar g^{\prime}\left(f_{0}\right) f_{1}+O\left(\hbar^{2}\right) .
$$

2.4. On the variation of $\tilde{S}_{0}$ and $\tilde{S}_{1}$. Let us deduce formulas (2.5) on the variations of $\tilde{S}_{0}$ and $\tilde{S}_{1}$ from the trace formula (2.8) and the functional symbolic calculus. Assume that $f_{0}^{-1}(I)$ is connected.

If $g \in C_{o}^{\infty}(I, \mathbb{C})$, then we deduce from $(2.8),(2.10)$ and the fact that $f_{1}=0$ that

$$
\begin{aligned}
\operatorname{Tr}\left(g\left(T_{k}\right)\right) & =\frac{k}{2 \pi} \int_{M}\left(g \circ f_{0}\right)\left(\omega+k^{-1} \omega_{1}\right)+O\left(k^{-1}\right) \\
& =\frac{k}{2 \pi} \int_{I} g\left(f_{0 *}\left[\omega+k^{-1} \omega_{1}\right]\right)+O\left(k^{-1}\right),
\end{aligned}
$$

where $f_{0 *}$ is the push-forward $\Omega^{2}(M) \rightarrow \Omega^{1}(I)$ defined by

$$
\int_{M}\left(f_{0}^{*} h\right) \alpha=\int_{I} g \cdot f_{0 *} \alpha, \quad \forall h \in C_{o}^{\infty}(I) .
$$

On the other hand, assume the Bohr-Sommerfeld condition is

$$
\tilde{S}(\lambda, k) \in 2 \pi k^{-1} \mathbb{Z}
$$

with $\tilde{S}(\lambda, k)=\tilde{S}_{0}+k^{-1} \tilde{S}_{1}+O\left(k^{-2}\right)$. If the derivative of $\tilde{S}_{0}$ does not vanish, then one can invert the functions $\tilde{S}(\cdot, k)$ when $k$ is sufficiently large and

$$
\operatorname{Tr}\left(g\left(T_{k}\right)\right)=\sum_{x \in 2 \pi k^{-1} \mathbb{Z}} g\left(\tilde{S}^{-1}(x, k)\right)+O\left(k^{-1}\right) .
$$


Interpreting this as a Riemann sum, it follows that

$$
\begin{aligned}
\operatorname{Tr}\left(g\left(T_{k}\right)\right) & =\frac{k}{2 \pi} \int_{\mathbb{R}} g\left(\tilde{S}^{-1}(x, k)\right) d x+O\left(k^{-1}\right) \\
& =\frac{k}{2 \pi} \int_{I} g(\lambda) \tilde{S}^{\prime}(\lambda, k) d \lambda+O\left(k^{-1}\right)
\end{aligned}
$$

with the same orientation of $I$ as before if $\tilde{S}_{0}^{\prime}$ is positive. Since (2.11) and (2.12) hold for any function $g$ of $C_{o}^{\infty}(I, \mathbb{C})$, we have

$$
d \tilde{S}_{0}+\hbar d \tilde{S}_{1}=f_{0 *}\left(\omega+\hbar \omega_{1}\right)
$$

and (2.5) follows.

\section{Lagrangian states}

3.1. Definitions and symbolic calculus. First we recall the definition of a local Lagrangian section associated to a closed Lagrangian embedding $\iota: \Gamma \rightarrow M$.

Let $U$ be an open set of $M$ such that $U_{\Gamma}:=\iota^{-1}(U)$ is contractible. Since the curvature of $\iota^{*} L$ vanishes, there exists a flat unitary section $t_{\Gamma}$ of $\iota^{*} L \rightarrow$ $U_{\Gamma}$. Introduce a formal series

$$
\sum_{\ell=0}^{\infty} \hbar^{\ell} g_{\ell} \in C^{\infty}\left(U_{\Gamma}, \iota^{*} K\right)[[\hbar]] .
$$

Let $V$ be an open set of $M$ such that $\bar{V} \subset U$. Then a sequence $\Psi_{k} \in \mathcal{H}_{k}$ is a Lagrangian section over $V$ associated to $\left(\Gamma, t_{\Gamma}\right)$ with symbol $\sum \hbar^{\ell} g_{\ell}$ if

$$
\Psi_{k}(x)=\left(\frac{k}{2 \pi}\right)^{n / 4} F^{k}(x) \tilde{g}(x, k)+O\left(k^{-\infty}\right) \text { over } V,
$$

where

- $F$ is a section of $L \rightarrow U$ such that

$$
\iota^{*} F=t_{\Gamma} \quad \text { and } \quad \bar{\partial} F \equiv 0
$$

modulo a section which vanishes to every order along $\iota(\Gamma)$. Furthermore, $|F(x)|<1$ if $x \notin \iota(\Gamma)$.

- $\tilde{g}(\cdot, k)$ is a sequence of $C^{\infty}(U, K)$ with an asymptotic expansion $\sum k^{-\ell} \tilde{g}_{\ell}$ in the $C^{\infty}$ topology such that

$$
\iota^{*} \tilde{g}_{\ell}=g_{\ell} \quad \text { and } \quad \bar{\partial} \tilde{g}_{\ell} \equiv 0
$$

modulo a section which vanishes at every order along $\iota(\Gamma)$.

We assume furthermore that $\Psi_{k}$ is admissible in the sense that $\Psi_{k}(x)$ is uniformly $O\left(k^{N}\right)$ for some $N$ and the same holds for its successive covariant derivatives.

It is not obvious that such a sequence exists. 
Theorem 3.1. For every series $\sum \hbar^{\ell} g_{\ell}$ of $C^{\infty}\left(U_{\Gamma}, \iota^{*} K\right)[[\hbar]]$, there exists a Lagrangian section over $V$ associated to $\left(\Gamma, t_{\Gamma}\right)$ with symbol $\sum \hbar^{\ell} g_{\ell}$. It is unique modulo a section which is $O\left(k^{-\infty}\right)$ over $V$.

In the statement of the following theorems, we consider that $K=L_{1} \otimes \delta$ over $U$, where $(\delta, \varphi)$ is a half-form bundle. Recall that $\iota^{*} \delta=\delta_{\Gamma}$ is a square root of $\Lambda^{n} T^{*} \Gamma \otimes \mathbb{C}$ through the isomorphism

$$
\varphi_{\Gamma}: \delta_{\Gamma}^{2} \longrightarrow \Lambda^{n} T^{*} \Gamma \otimes \mathbb{C}, \quad u \longrightarrow \iota^{*} \varphi(u) .
$$

Let us associate to the principal symbol $g_{0}$ of a Lagrangian section a density $m\left(g_{0}\right)$, where $m$ is the map:

$$
m: \iota^{*} L_{1} \otimes \delta_{\Gamma} \longrightarrow|\Lambda|(\Gamma), \quad u \otimes v \longrightarrow\|u\|_{L_{1}}^{2}\left|\varphi_{\Gamma}\left(v^{\otimes 2}\right)\right| .
$$

We then have the following estimate of the norm of $\Psi_{k}$.

Theorem 3.2. Let $\xi \in C_{o}^{\infty}(V)$. Then, we have

$$
\int_{M} \xi\left\|\Psi_{k}\right\|_{L^{k} \otimes L_{1} \otimes \delta}^{2} \mu_{M}=\int_{\Gamma}\left(\iota^{*} \xi\right) m\left(g_{0}\right)+O\left(k^{-1}\right) .
$$

Next results describe how a Toeplitz operator acts on a Lagrangian section.

Theorem 3.3. Let $T_{k}$ be a Toeplitz operator with principal symbol $f_{0}$. Then $T_{k} \Psi_{k}$ is a Lagrangian section over $V$ associated to $\left(\Gamma, t_{\Gamma}\right)$ with symbol $\left(\iota^{*} f_{0}\right) g_{0}+O(\hbar)$.

To prove the Bohr-Sommerfeld conditions, we need to compute the subsequent coefficient of the symbol of $T_{k} \Psi_{k}$, in the case where $f_{0}$ is constant over $\Gamma$.

Theorem 3.4. Let $T_{k}$ be a Toeplitz operator with normalized symbol $f_{0}+$ $\hbar f_{1}+O\left(\hbar^{2}\right)$. Assume that $f_{0}$ is constant along $\Gamma$. Then the symbol of $T_{k} \Psi_{k}$ is

$$
\left(\iota^{*}\left(f_{0}+\hbar f_{1}\right)\right)\left(g_{0}+\hbar g_{1}\right)+\hbar \frac{1}{i}\left(\nabla_{X}^{\iota^{*} L_{1}} \otimes \mathrm{Id}+\mathrm{Id} \otimes \mathcal{L}_{X}^{\delta_{\Gamma}}\right) g_{0}+O\left(\hbar^{2}\right),
$$

where

- $X$ is the Hamiltonian vector field of $f_{0}$,

- $\nabla^{\iota^{*} L_{1}}$ is the pull-back of the Chern connection of $L_{1}$,

- $\mathcal{L}_{X}^{\delta_{\Gamma}}$ is the first-order differential operator acting on sections of $\delta_{\Gamma}$ such that

$$
\varphi_{\Gamma}\left(\mathcal{L}_{X}^{\delta_{\Gamma}} g \otimes g\right)=\frac{1}{2} \mathcal{L}_{X} \varphi_{\Gamma}\left(g^{\otimes 2}\right)
$$

for every section $g$. 
It is easily checked that the operator $\nabla_{X}^{\iota^{*} L_{1}} \otimes \mathrm{Id}+\mathrm{Id} \otimes \mathcal{L}_{X}^{\delta_{\Gamma}}$ does not depend of the choice of the half-form bundle if we consider that it acts on sections of $\iota^{*} K=\iota^{*} L_{1} \otimes \delta_{\Gamma}$. The same holds with the map $m$.

Theorems 3.1, 3.2, 3.3 and 3.4 will be proven in Chapter 4, but first we will show how they imply Theorem 2.1 .

3.2. Proof of Bohr-Sommerfeld conditions. Let us deduce from the previous theorems the Bohr-Sommerfeld conditions for $n$ self-adjoint commuting Toeplitz operators $T^{1}, T^{2}, \ldots, T^{n}$, which is a slight generalization of (2.1).

Denote by $f_{0}^{i}$ and $f_{1}^{i}$ the principal and subprincipal symbols of $T^{i}$. Let $E$ be a regular value of $f=\left(f_{0}^{1}, \ldots, f_{0}^{n}\right)$ and $\iota: \Gamma \rightarrow M$ be an embedding with image a connected component of $f^{-1}(\{E\})$. Since $M$ is compact, $\iota(\Gamma)$ is a Lagrangian torus. So, there exists a half-form bundle $(\delta, \varphi)$ defined over a neighborhood of $\iota(\Gamma)$. It is not unique, but as usual, the final result does not depend on the choice of $(\delta, \varphi)$. Introduce like in the previous section two open sets $U, V$ and a flat section $t_{\Gamma}$. Let us try to solve the eigenvalues equation

$$
T^{i} \Psi=E^{i} \Psi+O\left(k^{-\infty}\right) \quad \text { over } V,
$$

where $\Psi$ is a Lagrangian section associated to $\left(\Gamma, t_{\Gamma}\right)$. By Theorem 3.4, the symbol of $\left(T^{i}-E^{i}\right) \Psi$ is $O(\hbar)$ because $\iota^{*} f^{i}=E^{i}$. Furthermore, it is $O\left(\hbar^{2}\right)$ if and only if it satisfies the following transport equation

$$
\left[f_{1}^{i}+\left(\nabla_{X^{i}}^{\iota^{*} L_{1}} \otimes \mathrm{Id}+\mathrm{Id} \otimes \mathcal{L}_{X^{i}}^{\delta_{\Gamma}}\right)\right] g_{0}=0 \text { over } V \cap \Gamma,
$$

where $g_{0}$ is the principal symbol of $\Psi$. This equation can be interpreted as $g_{0}$ being flat for a connection on $\iota^{*} L_{1} \otimes \delta_{\Gamma}$ that we describe now.

First a section $g$ of $\delta_{\Gamma}$ is flat if $\mathcal{L}_{X^{i}}^{\delta_{\Gamma}} g=0$ for every $i$. With this definition, $\varphi_{\Gamma}: \delta_{\Gamma}^{2} \rightarrow \Lambda^{n} T^{*} \Gamma \otimes \mathbb{C}$ is a morphism of flat bundles, if we endow $\Lambda^{n} T^{*} \Gamma \otimes \mathbb{C}$ with the Weinstein connection. Since the form $\beta$ such that

$$
\left\langle\beta, X^{1} \wedge \cdots \wedge X^{n}\right\rangle=1
$$

is a global non-vanishing flat section of $\Lambda^{n} T^{*} \Gamma \otimes \mathbb{C}, \delta_{\Gamma}$ has holonomy in $\mathbb{Z}_{2} \subset U(1)$.

Let $\alpha \in \Omega^{1}(\Gamma)$ be such that $\frac{1}{i}\left\langle\alpha, X^{j}\right\rangle=f_{1}^{j}$. Consider the connection $\nabla^{\iota^{*} L_{1}}+\alpha$ on $\iota^{*} L_{1}$. Its flat sections satisfy

$$
\left(f_{1}^{j}+\frac{1}{i} \nabla_{X^{j}}^{\iota^{*} L_{1}}\right) s=0
$$

Furthermore, its curvature vanishes. Indeed, since $\left[X^{i}, X^{j}\right]=0$, we have

$$
\begin{aligned}
\frac{1}{i}\left\langle d \alpha, X^{i} \wedge X^{j}\right\rangle & =\left(X^{i} \cdot f_{1}^{j}-X^{j} \cdot f_{1}^{i}\right) \\
& =\omega_{1}\left(X^{i}, X^{j}\right)
\end{aligned}
$$

which follows from Theorem 1.4 and the fact that $\left[T^{i}, T^{j}\right]=0$. 
This defines a structure of flat line bundle for $\iota^{*} L_{1} \otimes \delta_{\Gamma}$, whose flat sections are the solutions of (3.2). Recall that

$$
\Psi(x, k)=t_{\Gamma}^{k}(x)\left(g_{0}(x)+O\left(k^{-1}\right)\right) \quad \text { over } V \cap \iota(\Gamma),
$$

where $t_{\Gamma}$ is a flat section of $\iota^{*} L$. The condition to patch together these sections along $\Gamma$ is the Bohr-Sommerfeld condition:

$$
\iota^{*}\left(L^{k} \otimes L_{1}\right) \otimes \delta_{\Gamma} \longrightarrow \Gamma \text { is trivial as a flat bundle. }
$$

When $M$ is two-dimensional, this is equivalent to (2.1). This completes the proof of Theorem 2.1, except for the analytical aspects. Actually we should consider families of Lagrangian sections depending continuously on $\Gamma$ and prove that the solutions of (3.1) are necessarily Lagrangian sections associated to $\Gamma$. A complete proof is in [7]. The only novelty here is the geometrical formulation of Theorems 3.4 and 3.2 and consequently of the Bohr-Sommerfeld conditions.

3.3. Comparison with the cotangent case. To compare Theorems 3.2 and 3.4 with the similar statements in the case of pseudo-differential operators, we can introduce some kind of Maslov bundle in the following way. Recall that we denote by $\varphi_{\Gamma}$ the isomorphism $\delta_{\Gamma}^{2} \rightarrow \Lambda^{n} T^{*} \Gamma \otimes \mathbb{C}$. Introduce

$$
P:=\left\{u \in \delta_{\Gamma} ; \varphi_{\Gamma}\left(u^{\otimes 2}\right) \in \Lambda^{n} T^{*} \Gamma-\{0\}\right\} .
$$

Let $\mathbb{Z}_{4}$ be the subgroup $\{1,-1, i,-i\}$ of $\mathbb{C}^{*}$. Then $P$ is a principal bundle with structure group $\mathbb{Z}_{4} \times \mathbb{R}_{+}$. Introduce the complex line bundles $\left|\delta_{\Gamma}\right|$ and $\arg \left(\delta_{\Gamma}\right)$ associated to $P$ via the homomorphism $\mathbb{Z}_{4} \times \mathbb{R}_{+} \rightarrow \mathbb{Z}_{4}$ and $\mathbb{Z}_{4} \times \mathbb{R}_{+} \rightarrow \mathbb{R}_{+}$, respectively. Following Weinstein in $[\mathbf{2 1}]$, we call $\arg \left(\delta_{\Gamma}\right)$ the unitarization of $\delta_{\Gamma}$. We have a canonical isomorphism

$$
\delta_{\Gamma} \longrightarrow\left|\delta_{\Gamma}\right| \otimes \arg \left(\delta_{\Gamma}\right) .
$$

Furthermore, the map

$$
\left|\delta_{\Gamma}\right|=P \times_{\mathbb{R}_{+}} \mathbb{C} \ni[u, z] \longrightarrow z \cdot\left|\varphi_{\Gamma}\left(u^{\otimes 2}\right)\right|^{1 / 2} \in|\Lambda|^{1 / 2}(\Gamma)
$$

is an isomorphism between $\left|\delta_{\Gamma}\right|$ and the bundle of half-densities of $\Gamma$. So we obtain an isomorphism

$$
\zeta: \delta_{\Gamma} \longrightarrow|\Lambda|^{1 / 2}(\Gamma) \otimes \arg \left(\delta_{\Gamma}\right)
$$

The bundle $\arg \left(\delta_{\Gamma}\right)$ is a line bundle with structure group $\mathbb{Z}_{4}$ like the Maslov bundle. The isomorphism $\zeta$ intertwines the operator $\mathcal{L}_{X}^{\delta_{\Gamma}}$ of Theorem 3.4 with the Lie derivative of half-densities. So, in the case $L_{1}$ is trivial, Theorem 3.4 is similar to formula 1.3 .13 in [10, p. 223], computing the symbol of an oscillatory integral acted on under a differential operator. Furthermore, the map $\delta_{\Gamma} \rightarrow|\Lambda|(\Gamma)$ used in Theorem 3.2 is the composition of $\zeta$ with the squaring map from half-densities into densities. Again Theorem 3.2 is similar to formula 1.3 .15 in [10, p.224], computing the norm of an oscillatory integral. 
To end this comparison, we apply the previous construction to a symplectic vector space $E$ and prove that we obtain the usual Maslov bundle. Consider a one-dimensional vector space $\delta$ with an isomorphism $\varphi: \delta^{2} \rightarrow \Lambda^{n, 0} E^{*}$. Let $\operatorname{Lag}(E)$ be the Lagrangian Grassmannian and $\eta \rightarrow \operatorname{Lag}(E)$ be the tautological vector bundle, that is the bundle whose fiber over $x$ is $x$ itself. In the same way we defined $\varphi_{\Gamma}$, one has an isomorphism

$$
\varphi_{\text {Lag }}: \operatorname{Lag}(E) \times \delta^{2} \longrightarrow \Lambda^{n} \eta \otimes \mathbb{C},
$$

sending $(x, u)$ into $\iota_{x}^{*} \varphi(u)$, where $\iota_{x}$ is the embedding $\eta_{x} \rightarrow E$. Introduce the $\mathbb{Z}_{4} \times \mathbb{R}_{+}$bundle

$$
\left\{(x, u) \in \operatorname{Lag}(E) \times \delta ; \varphi_{\operatorname{Lag}}\left(x, u^{2}\right) \in \Lambda_{x}^{n} \eta \text { and } u \neq 0\right\} .
$$

Dividing by $\mathbb{R}_{+}$, we get a $\mathbb{Z}_{4}$-principal bundle $\mathcal{M}$. We claim that the holonomy in $\mathcal{M}$ of a loop of $\operatorname{Lag}(E)$ is the mod 4 reduction of its Maslov index.

Proof. Introduce linear Darboux coordinates $\left(p^{i}, q^{i}\right)$ and identify $E$ with $\mathbb{R}^{2 n}$. Set $z^{j}=p^{j}+i q^{j}$ and let

$$
\delta:=\left(d z^{1} \wedge \cdots \wedge d z^{n}\right)^{1 / 2} \mathbb{C}
$$

be the square root of $\Lambda^{n, 0} E=\left(d z^{1} \wedge \cdots \wedge d z^{n}\right) \mathbb{C}$. Recall that $\operatorname{Lag}(E)$ is isomorphic to $U(n) / O(n)$ (cf. Lemma 2.31 of [17]) through the map sending the unitary matrix $U=P+i Q$ into the range of

$$
A_{U}=\left(\begin{array}{c}
P \\
Q
\end{array}\right) \text {. }
$$

Let us denote by $\alpha_{U}^{1}, \ldots, \alpha_{U}^{n}$ the base of $\eta_{x}^{*}$ dual to the column vectors of $A_{U}$. A straightforward computation shows that $\varphi_{\text {Lag }}$ sends the square of

$$
\left([U],\left(d z^{1} \wedge \cdots \wedge d z^{n}\right)^{1 / 2}\right) \in \operatorname{Lag}(E) \times \delta
$$

into $\operatorname{det}(U) \alpha_{U}^{1} \wedge \cdots \wedge \alpha_{U}^{n}$. Consequently,

$$
\mathcal{M} \simeq\left\{([U], v) \in \operatorname{Lag}(E) \times \mathbb{C}^{*} ; U \in U(n) \text { and } v^{2} \operatorname{det}(U)= \pm 1\right\} .
$$

Recall now that the Maslov index of a loop $x: \mathbb{R} / \mathbb{Z} \rightarrow \operatorname{Lag}(E)$ is the degree of $\rho \circ x: S^{1} \rightarrow S^{1}$, where $\rho$ is the map

$$
\operatorname{Lag}(E) \longrightarrow S^{1}, \quad[U] \longrightarrow \operatorname{det}^{2}(U)
$$

(cf. [17], page 53). Its mod 4 reduction is the holonomy of $x$ in $\mathcal{M}$.

This result is related to the paper [21] of Weinstein, where it is observed that the Maslov bundle of $\operatorname{Lag}(E)$ is a unitarization of a square root of $\Lambda^{n} \eta \otimes \mathbb{C}$.

Last remark is that, in general, the Maslov bundle of a Lagrangian submanifold of a cotangent space can be different from the bundle we construct. Indeed, notice that the structure group of $\arg \left(\delta_{\Gamma}\right)$ reduces to $\mathbb{Z}_{2}$ if and only if $\Gamma$ is orientable. Consider a non-orientable manifold $Q$. Then, as the null 
section of $T^{*} Q, Q$ is a Lagrangian submanifold and its Maslov bundle is the flat trivial bundle, so it cannot be a unitarization of a square root of $\Lambda^{n} T^{*} Q \otimes \mathbb{C}$.

\section{Proof}

We assume in the whole section that there exists a globally defined halfform bundle $(\delta, \varphi)$ and $K=\delta$. There is no difficulty to generalize to the case where $K=\delta \otimes L_{1}$. Next section contains preliminaries results leading to the proof of the theorems of part 3.1.

4.1. A preliminary result. Consider a sequence $\Psi_{k} \in C^{\infty}\left(M, L^{k} \otimes \delta\right)$ of the form

$$
\Psi_{k}(x)=\left(\frac{k}{2 \pi}\right)^{n / 4} F^{k}(x) \tilde{g}(x, k)+O\left(k^{-\infty}\right) \text { over } V,
$$

where $F$ and $\tilde{g}(\cdot, k)$ satisfies the same assumptions as in Section 3.1, except that the coefficients $\tilde{g}_{k}$ do not necessarily satisfy $\bar{\partial} \tilde{g}_{k} \equiv 0$. Assume furthermore that $\Psi_{k}$ is admissible.

Theorem 4.1. Let $T_{k}$ be a Toeplitz operator with principal symbol $f_{0}$. Then $T_{k} \Psi_{k}$ is a Lagrangian section over $V$ with symbol $\iota^{*}\left(f_{0} \tilde{g}_{0}\right)+O(\hbar)$.

Furthermore, if $\tilde{g}_{0}$ and its first derivatives vanish along $\iota(\Gamma)$, then the symbol of $T_{k} \Psi_{k}$ is

$$
\left.\hbar\left(\iota^{*} f_{0}\right)\left(\square \tilde{g}_{0}+\iota^{*} \tilde{g}_{1}\right)\right)+O\left(\hbar^{2}\right)
$$

where $\square \tilde{g}_{0} \in C^{\infty}\left(\Gamma, \delta_{\Gamma}\right)$ and at every $x \in \Gamma$

$$
\square \tilde{g}_{0}(x)=-\frac{1}{2} \sum \bar{\partial}_{i} \bar{\partial}_{i} \tilde{g}_{0}(\iota(x))
$$

if $\partial_{1}, \ldots, \partial_{n}$ is a base of vectors of $T_{\iota(x)}^{1,0} M$ such that $\frac{1}{i} \omega\left(\partial_{i}, \bar{\partial}_{j}\right)=\delta_{i j}$ and the vectors $\partial_{i}+\bar{\partial}_{i}$ are tangent to $\Gamma$.

The proof starts from the following representation of the Schwartz kernel of the Toeplitz operator $T_{k}$ :

$$
T_{k}(x, y)=\left(\frac{k}{2 \pi}\right)^{n} E^{k}(x, y) \tilde{f}(x, y, k)+O\left(k^{-\infty}\right),
$$

where, if we consider $M^{2}$ as a complex manifold with complex structure $(j,-j)$,

- $E$ is a section of $L \otimes \bar{L} \rightarrow M^{2}$ satisfying

$$
E(x, x)=u \otimes \bar{u}, \quad \forall u \in L_{x} \text { such that }\|u\|=1,
$$

$\bar{\partial} E \equiv 0$ modulo a section vanishing to every order along the diagonal $\Delta$ of $M^{2}$ and $\|E(x, y)\|<1$ if $x \neq y$. 
- $\tilde{f}(\cdot, k)$ is a sequence of sections of $\delta \otimes \bar{\delta} \rightarrow M^{2}$ with an asymptotic expansion of the form

$$
\tilde{f}(\cdot, k)=\tilde{f}_{0}+k^{-1} \tilde{f}_{1}+\cdots
$$

whose coefficient satisfies $\bar{\partial} f_{l} \equiv 0$ modulo a section vanishing to every order along $\Delta$. Furthermore,

$$
\tilde{f}_{0}(x, x)=f_{0}(x),
$$

where $f_{0}$ is the principal symbol of $T_{k}$.

In other words, $T_{k}(\cdot, \cdot)$ is a Lagrangian section associated to the diagonal $\Delta$ of $M^{2}$. This result was proved in [6], without the additional bundle $\delta$. The generalization is straightforward.

Since the norm of $E$ is $<1$ outside the diagonal and $\Psi_{k}$ is admissible, one has for every $x$ in $V$

$$
\left(T_{k} \Psi_{k}\right)(x)=\left(\frac{k}{2 \pi}\right)^{n+(n / 4)} \int E^{k}(x, y) \cdot F^{k}(y) \tilde{f}(x, y, k) \cdot \tilde{g}(y, k) \mu_{M}(y)+O\left(k^{-\infty}\right),
$$

where we integrate on a neighborhood of $x$. Introduce a unitary section $t$ of $L$ over $U$ such that $\iota^{*} t=t_{\Gamma}$ over $U_{\Gamma}$ and let us write

$$
E(x, y) \cdot F(y)=\mathrm{e}^{i \phi(x, y)} t(x) .
$$

Then the imaginary part of $\phi$ is non-positive and vanishes only if $(x, y)$ belongs to

$$
C:=\left\{(x, x) \in M^{2} ; x \in \iota(\Gamma)\right\} .
$$

To compute the derivatives of $\phi$ along $C$, recall the following lemma proved in [7, cf. Proposition 2.2, p. 1535].

Lemma 4.2. If $\nabla^{L} F=\frac{1}{i} \alpha_{F} \otimes F$, then $\alpha_{F}$ vanishes along $\iota(\Gamma)$, and for every vector field $X, Y$

$$
\mathcal{L}_{X}\left\langle\alpha_{F}, Y\right\rangle=\omega(q X, Y)
$$

at $x \in \iota(\Gamma)$, where $q$ is the projection onto $T_{x}^{0,1} M$ with kernel $T_{x} \iota(\Gamma) \otimes \mathbb{C}$.

As a corollary, we have the following

Lemma 4.3. If $\nabla^{L \otimes \bar{L}} E=\frac{1}{i} \alpha_{E} \otimes E$, then $\alpha_{E}$ vanishes along the diagonal, and for every vector fields $X_{1}, Y_{1}, X_{2}, Y_{2}$ of $M$,

$$
\mathcal{L}_{\left(X_{1}, Y_{1}\right)} \cdot\left\langle\alpha_{E},\left(X_{2}, Y_{2}\right)\right\rangle=\omega\left(X_{1}^{0,1}-Y_{1}^{0,1}, X_{2}\right)+\omega\left(X_{1}^{1,0}-Y_{1}^{1,0}, Y_{2}\right)
$$

along the diagonal, where we denote by $X^{1,0}$ and $X^{0,1}$ the holomorphic and anti-holomorphic parts of $X$, respectively.

We deduce from both lemmas that $d_{y} \phi$ vanishes along $C$. Furthermore, the kernel of the tangent map to $d_{y} \phi$ at $(x, x) \in C$ is

$$
\left(T_{x}^{0,1} M \times(0)\right) \oplus\left(T_{(x, x)} C \otimes \mathbb{C}\right) .
$$


Finally, we have along $C$,

$$
d_{y}^{2} \phi\left(Y_{1}, Y_{2}\right)=\omega\left(Y_{1}^{1,0}, Y_{2}\right)-\omega\left(q Y_{1}, Y_{2}\right)
$$

and $d_{y}^{2} \phi$ is non-degenerate. So we can apply the stationary phase lemma (cf. [13], Section 7.7 or Theorem A.1). One gets

$$
\left(T_{k} \Psi_{k}\right)(x)=\left(\frac{k}{2 \pi}\right)^{n / 4} \mathrm{e}^{i k \phi_{r}(x)} t^{k}(x) \tilde{h}(x, k)+O\left(k^{-\infty}\right),
$$

where

$$
\phi_{r}(x) \equiv \phi(x, y)
$$

modulo a linear combination with $C^{\infty}$ coefficients of the $\partial_{y^{i}} \phi(x, y)$.

Lemma 4.4. $\mathrm{e}^{i k \phi_{r}(x)} t(x)$ satisfies the same assumption as the section $F$.

Proof. Since $\phi$ and $d_{y} \phi$ vanish along $C, \phi_{r}$ vanishes along $\iota(\Gamma)$ and consequently

$$
\mathrm{e}^{i \phi_{r}(x)} t(x)=t_{\Gamma}(x)
$$

for every $x$ in $\iota(\Gamma)$. Introduce complex coordinates $x^{1}, \ldots, x^{n}$ and write

$$
\nabla t=\frac{1}{i} t \otimes \sum a_{j}(x) d x^{j}+\bar{a}_{j}(x) d \bar{x}^{j} .
$$

Differentiating (4.2), it follows from $\bar{\partial} E \equiv 0$ that

$$
\partial_{\bar{x}^{i}} \phi(x, y) \equiv \bar{a}_{j}(x) \quad \bmod \mathcal{I}_{\Delta}(\infty),
$$

i.e., modulo a function vanishing to infinite order along the diagonal. Differentiating again, one has

$$
\partial_{\bar{x}^{i}} \partial_{y^{j}} \phi(x, y) \equiv 0 \quad \bmod \mathcal{I}_{\Delta}(\infty) .
$$

Then, we deduce from the two previous equations and (4.3) that for every multi-index $\alpha$,

$$
\partial_{\bar{x}^{1}}^{\alpha(1)} \cdots \partial_{\bar{x}^{n}}^{\alpha(n)}\left(\partial_{\bar{x}^{i}} \phi_{r}-\bar{a}_{i}\right)(x)=0
$$

along $\iota(\Gamma)$. And consequently

$$
\partial_{\bar{x}^{i}} \phi_{r} \equiv \bar{a}_{i} \quad \bmod \mathcal{I}_{\iota(\Gamma)}(\infty),
$$

which proves the result.

Lemma 4.5. The sequence $\tilde{h}(\cdot, k)$ admits an asymptotic expansion $\tilde{h}_{0}+$ $k^{-1} \tilde{h}_{1}+\cdots$ whose coefficients satisfy $\bar{\partial} \tilde{h}_{\ell} \equiv 0$ modulo a section vanishing to every order along $\Gamma$.

Proof. First, one deduces from Lemma 4.2 that the imaginary part of $\phi_{r}$ and its first derivatives vanish along $\iota(\Gamma)$. Furthermore, the Hessian of $\Im \phi_{r}$ along 
$\iota(\Gamma)$ is non-degenerate in the transverse direction to $\iota(\Gamma)$. As a consequence, if $\tilde{e}(x, k)$ is a sequence with an asymptotic expansion $\tilde{e}_{0}+k^{-1} \tilde{e}_{1}+\cdots$ such that

$$
\mathrm{e}^{i k \phi_{r}(x)} \tilde{e}(x, k)=O\left(k^{-\infty}\right),
$$

then the coefficients $\tilde{h}_{\ell}$ vanish to every order along $\iota(\Gamma)$. This was proved in $\left[\mathbf{6}\right.$, lemma 1 , p. 6]. We apply this to the sequence $\bar{\partial} T_{k} \Psi_{k}$ which vanishes, since $\Pi_{k} T_{k}=T_{k}$ implies that $T_{k} \Psi_{k}$ belongs to $\mathcal{H}_{k}$.

The two previous lemmas imply that $T_{k} \Psi_{k}$ is a Lagrangian section. Then applying Theorems A.1 and A.2, we obtain the symbol of $T_{k} \Psi_{k}$ by computations of linear algebra, which are easily done using the tangent vectors $\partial_{i}$ introduced in the statement of Theorem 4.1.

4.2. Proofs of the theorems of part 3.1. A first corollary of theorem 4.1 is the existence of a Lagrangian section with an arbitrary symbol: applying Theorem 4.1 with the Toeplitz operator $\Pi_{k}$, we construct a Lagrangian section with a prescribed principal symbol, and then Theorem 3.1 follows from Borel resummation. Theorem 3.3 is a particular case of Theorem 4.1.

To prove Theorem 3.4, we can assume that $f_{0}$ vanishes along $\Gamma$. Since we compute the symbol of $T_{k} \Psi_{k}$ modulo $O\left(\hbar^{2}\right)$, we can replace $T_{k}$ with every Toeplitz operators of symbol $f_{0}+\hbar f_{1}+O\left(\hbar^{2}\right)$. So by Theorem 1.5 , we can choose

$$
T_{k}=\Pi_{k}\left(f_{0}+k^{-1} f_{1}+\frac{1}{i k}\left(\nabla_{X}^{L^{k}} \otimes \mathrm{Id}+\mathrm{Id} \otimes D_{X}\right)\right),
$$

where $X$ is the Hamiltonian vector field of $f_{0}$. So $T_{k} \Psi_{k}$ is equal to

$$
\begin{aligned}
\Pi_{k}\left[\left(\frac{k}{2 \pi}\right)^{n / 4}\left(\left[f_{0}+\frac{1}{i k} \nabla_{X}^{L^{k}}\right] F^{k}\right) \tilde{g}(\cdot, k)\right] \\
\quad+k^{-1} \Pi_{k}\left[\left(\frac{k}{2 \pi}\right)^{n / 4} F^{k}\left(\left[f_{1}+\frac{1}{i} D_{X}\right] \tilde{g}(\cdot, k)\right)\right]
\end{aligned}
$$

By Theorem 4.1, each term of the sum is a Lagrangian section. Furthermore, by the first part of this theorem, the symbol of the second one is

$$
\hbar\left(\left(\iota^{*} f_{1}\right) \cdot g_{0}+\iota^{*}\left(\frac{1}{i} D_{X} \tilde{g}_{0}\right)\right)+O\left(\hbar^{2}\right) .
$$

Since $X$ is tangent to $\iota(\Gamma), \iota^{*}\left(D_{X} \tilde{g}_{0}\right)$ only depends on the restriction of $\tilde{g}_{0}$ to $\iota(\Gamma)$. So we can define the operator $\iota^{*} D_{X}$ acting on $C^{\infty}\left(\Gamma, \delta_{\Gamma}\right)$ which sends $g_{0}$ to $\iota^{*}\left(D_{X} \tilde{g}_{0}\right)$, and the previous symbol is

$$
\hbar\left(\left(\iota^{*} f_{1}\right)+\frac{1}{i}\left(\iota^{*} D_{X}\right)\right) \cdot g_{0}+O\left(\hbar^{2}\right) .
$$


To compute the symbol of the first term of (4.4), let us write

$$
\left[f_{0}+\frac{1}{i k} \nabla_{X}^{L^{k}}\right] F^{k}=F^{k} a
$$

with $a$ defined on a neighborhood of $\iota(\Gamma)$.

Lemma 4.6. The function $a$ and its first derivatives vanish along $\iota(\Gamma)$. If $Z$ and $W$ are holomorphic vector fields of $M$, then

$$
\mathcal{L}_{\bar{W}} \mathcal{L}_{\bar{Z}} a=\omega\left(\bar{W}, \mathcal{L}_{X} \bar{Z}\right)
$$

on $\iota(\Gamma)$.

Proof. Denote by $\alpha_{F}$ the one-form such that $\nabla^{L} F=\frac{1}{i} \alpha_{F} \otimes F$. Then,

$$
a=f_{0}-\left\langle\alpha_{F}, X\right\rangle \text {. }
$$

This vanishes along $\iota(\Gamma)$ because $\alpha_{F}$ vanishes along $\iota(\Gamma)$ (cf. Lemma 4.2).

Since $X$ is the Hamiltonian vector field of $f_{0}$, one has $\mathcal{L}_{Y} f_{0}+\omega(X, Y)=0$. Since the curvature of $L$ is $\frac{1}{i} \omega$, one has $d \alpha_{F}=\omega$ and consequently

$$
\mathcal{L}_{Y}\left\langle\alpha_{F}, X\right\rangle=\mathcal{L}_{X}\left\langle\alpha_{F}, Y\right\rangle+\omega(Y, X)+\left\langle\alpha_{F},[Y, X]\right\rangle .
$$

It follows that

$$
\mathcal{L}_{Y} a=-\mathcal{L}_{X}\left\langle\alpha_{F}, Y\right\rangle-\left\langle\alpha_{F},[Y, X]\right\rangle .
$$

This vanishes along $\iota(\Gamma)$ because $\alpha_{F}$ vanishes along $\iota(\Gamma)$ and $X$ is tangent to $\iota(\Gamma)$.

Since $\bar{\partial} F \equiv 0$ modulo a flat section and $Z$ is holomorphic, $\left\langle\alpha_{F}, \bar{Z}\right\rangle$ vanishes to every order along $\iota(\Gamma)$. So choosing $Y=\bar{Z}$ in the previous equation, we obtain

$$
\mathcal{L}_{\bar{W}} \cdot \mathcal{L}_{\bar{Z}} a=-\mathcal{L}_{\bar{W}}\left\langle\alpha_{F},[\bar{Z}, X]\right\rangle \quad \text { along } \iota(\Gamma) .
$$

Using again that $\omega=d \alpha_{F}$ and $\alpha_{F}$ vanishes along $\iota(\Gamma)$, it follows that

$$
\mathcal{L}_{\bar{W}} \cdot \mathcal{L}_{\bar{Z}} a=-\omega(\bar{W},[\bar{Z}, X])-\mathcal{L}_{[\bar{Z}, X]}\left\langle\alpha_{F}, \bar{W}\right\rangle
$$

along $\iota(\Gamma)$. The second term of the right side vanishes along $\iota(\Gamma)$ because $\bar{W}$ is an anti-holomorphic vector field. This gives the result.

Since $\iota^{*} D_{X}$ and $\mathcal{L}_{X}^{\delta_{\Gamma}}$ are first-order differential operators which have the same symbol,

where $b$ is a function of $\Gamma$.

$$
\iota^{*} D_{X}-\mathcal{L}_{X}^{\delta_{\Gamma}}=b
$$

Lemma 4.7. The symbol of $\Pi_{k}\left[F^{k} a \tilde{g}(\cdot, k)\right]$ is $i \hbar b . g_{0}+O\left(\hbar^{2}\right)$.

So, the symbol of $T_{k} \Psi_{k}$ is the sum of (4.5) and $i \hbar b g_{0}+O\left(\hbar^{2}\right)$, which is equal to

Theorem 3.4 follows.

$$
\hbar\left(\iota^{*} f_{1}+\frac{1}{i} \mathcal{L}_{X}^{\delta_{\Gamma}}\right) \cdot g_{0}+O\left(\hbar^{2}\right)
$$


Proof. Let us start with a local computation of the function $b$. Let $u$ be a non-vanishing section of $\delta_{\Gamma} \rightarrow \Gamma$. Then, one has

$$
b=\frac{\left(\iota^{*} D_{X}-\mathcal{L}_{X}^{\delta_{\Gamma}}\right) \cdot u}{u} .
$$

Let $\beta$ be a non-vanishing $(n, 0)$-form of $M$ such that $\varphi\left(u^{\otimes 2}(x)\right)=\beta(\iota(x))$ if $x$ belongs to $\Gamma$. Then, $\iota^{*} D_{X}$ is defined in such a way that

$$
\frac{\left(\iota^{*} D_{X}\right) \cdot u}{u}=\frac{1}{2} \iota^{*}\left(\frac{p \mathcal{L}_{X} \beta}{\beta}\right)=\frac{1}{2} \frac{\iota^{*}\left(p \mathcal{L}_{X} \beta\right)}{\iota^{*} \beta},
$$

where $p$ is the projection from $\Lambda^{n} M \otimes \mathbb{C}$ onto $\Lambda^{n, 0} M$ with kernel $\Lambda^{0, n} M \oplus$ $\cdots \oplus \Lambda^{n-1,1} M$. On the other hand, since $\varphi_{\Gamma}\left(u^{\otimes 2}(x)\right)=\iota^{*} \varphi\left(u^{\otimes 2}(x)\right)=\iota^{*} \beta(x)$, one has

$$
\frac{\mathcal{L}_{X}^{\delta_{\Gamma}} \cdot u}{u}=\frac{1}{2} \frac{\mathcal{L}_{X} \iota^{*} \beta}{\iota^{*} \beta}=\frac{1}{2} \frac{\iota^{*} \mathcal{L}_{X} \beta}{\iota^{*} \beta} .
$$

Consequently,

$$
b=\frac{1}{2} \frac{\iota^{*}\left(p \mathcal{L}_{X} \beta-\mathcal{L}_{X} \beta\right)}{\iota^{*} \beta} .
$$

Now let us choose a frame $\left(\partial_{1}, \ldots, \partial_{n}\right)$ of holomorphic vector fields of $M$ such that the vectors $\partial_{i}+\bar{\partial}_{i}$ are tangent to $\iota(\Gamma)$. Denote by $\left(\theta^{1}, \ldots, \theta^{n}\right)$ the dual frame and set

$$
\beta=\theta^{1} \wedge \cdots \wedge \theta^{n}
$$

Then, using that $\mathcal{L}_{X} \theta^{i} \equiv-\sum\left\langle\theta^{i}, \mathcal{L}_{X} \bar{\partial}_{j}\right\rangle \bar{\theta}^{j}$ modulo a linear combination of the $\theta^{i}$, we obtain that $\iota^{*}\left(p \mathcal{L}_{X} \beta-\mathcal{L}_{X} \beta\right)$ is equal to

$$
\begin{gathered}
\sum\left\langle\theta^{1}, \mathcal{L}_{X} \bar{\partial}_{j}\right\rangle \bar{\theta}^{j} \wedge \theta^{2} \wedge \cdots \wedge \theta^{n}+\left\langle\theta^{2}, \mathcal{L}_{X} \bar{\partial}_{j}\right\rangle \theta^{1} \wedge \bar{\theta}^{j} \wedge \theta^{3} \wedge \cdots \wedge \theta^{n}+\cdots \\
+\left\langle\theta^{n}, \mathcal{L}_{X} \bar{\partial}_{j}\right\rangle \theta^{1} \wedge \cdots \wedge \theta^{n-1} \wedge \bar{\theta}^{j}
\end{gathered}
$$

It follows then from $\iota^{*} \theta^{i}=\iota^{*} \bar{\theta}^{i}$ that

$$
b=\frac{1}{2} \sum\left\langle\theta^{i}, \mathcal{L}_{X} \bar{\partial}_{i}\right\rangle .
$$

To end the proof, assume furthermore that $\frac{1}{i} \omega\left(\partial_{i}, \bar{\partial}_{j}\right)=\delta_{i j}$. Then, it follows from the second part of Theorem 4.1 that the symbol of $\Pi_{k}\left[F^{k} a \tilde{g}(\cdot, k)\right]$ is

$$
-\hbar g_{0} \frac{1}{2} \iota^{*} \sum \mathcal{L}_{\bar{\partial}_{i}} \mathcal{L}_{\bar{\partial}_{i}} a
$$

which by Lemma 4.6 is equal to

$$
-\hbar g_{0} \frac{1}{2} \iota^{*} \sum \omega\left(\bar{\partial}_{i}, \mathcal{L}_{X} \bar{\partial}_{i}\right)
$$

Using again that $\frac{1}{i} \omega\left(\partial_{i}, \bar{\partial}_{j}\right)=\delta_{i j}$, we obtain

$$
\hbar g_{0} \frac{i}{2} \iota^{*} \sum\left\langle\theta^{i}, \mathcal{L}_{X} \bar{\partial}_{i}\right\rangle .
$$

The final result follows. 
Finally, let us prove Theorem 3.2. One has

$$
\int_{M} \xi\left\|\Psi_{k}\right\|_{L^{k} \otimes \delta}^{2} \mu_{M}=\left(\frac{k}{2 \pi}\right)^{n / 2} \int_{M} \mathrm{e}^{-k c} \xi\|\tilde{g}(\cdot, k)\|_{\delta}^{2} \mu_{M}+O\left(k^{-\infty}\right),
$$

where $c(x)=-2 \ln \|F(x)\|_{L}$. The following is a consequence of Lemma 4.2.

Lemma 4.8. The function $c$ and its first derivatives vanish along $\iota(\Gamma)$. Furthermore, its Hessian at $x \in \iota(\Gamma)$ is definite positive on $J T_{x} \iota(\Gamma)$ and is given by

$$
X \cdot Y \cdot c=2 \omega(X, J Y), \quad X, Y \in J T_{x} \iota(\Gamma) .
$$

So, integrating along transversal directions to $\Gamma$, it follows from the stationary phase lemma that

$$
\int_{M} \xi\left\|\Psi_{k}\right\|_{L^{k} \otimes \delta}^{2} \mu_{M}=\int_{\Gamma}\left(\iota^{*} \xi\right) d+O\left(k^{-1}\right),
$$

where $d$ is the density of $\Gamma$ such that

$$
\left.d\right|_{x}(X)=\left.\left\|g_{0}(x)\right\|_{\delta}^{2} \mu_{M}\right|_{x}(X \wedge Y) 2^{-n / 2}\left(\operatorname{det}\left[\omega\left(Y_{i}, J Y_{j}\right)\right]\right)^{-1 / 2} .
$$

Here $\left(X_{i}\right)$ and $\left(Y_{i}\right)$ are bases of $T_{x} \Gamma$ and $J T_{x} \Gamma$, respectively, and $X=X_{1} \wedge$ $\cdots \wedge X_{n}, Y=Y_{1} \wedge \cdots \wedge Y_{n}$. To deduce Theorem 3.2, we have to check that

$$
d=m\left(g_{0}\right) \text {. }
$$

This is easily done by introducing the same vector fields $\partial_{i}$ and forms $\theta^{i}$ like in the proof of Lemma 4.7, setting $X_{i}=\frac{1}{\sqrt{2}}\left(\partial_{i}+\bar{\partial}_{i}\right), Y_{j}=J X_{j}$ and choosing $g_{0}$ such that $\varphi\left(g_{0}^{\otimes 2}\right)=\theta_{1} \wedge \cdots \wedge \theta^{n}$.

\section{Appendix A.}

Let $W$ be an open set of $\mathbb{R}^{n} \times \mathbb{R}^{k} \ni(x, y)$. Denote by $p$ the projection from $W$ onto $\mathbb{R}^{n}$. Let $\varphi(x, y)$ be a $C^{\infty}$ function on $W$ whose imaginary part is $\geqslant 0$. Let $a(x, y)$ be a $C^{\infty}$ function with compact support in $W$. Stationary phase lemma gives the asymptotic expansion of

$$
I(a, \varphi)(x, \tau)=\int_{\mathbb{R}^{k}} \mathrm{e}^{i \tau \varphi(x, y)} a(x, y)|d y|
$$

when $\tau \rightarrow \infty$. First, if the support of $a$ does not meet the critical locus

$$
C:=\left\{(x, y) \in W ; d_{y} \varphi(x, y)=0 \text { and } \Im \varphi(x, y)=0\right\},
$$

then $I(a, \varphi)$ is $O\left(\tau^{-\infty}\right)$. Introduce the functions

$$
\varphi_{i, j}(x, y)=\partial_{y^{i}} \partial_{y^{j}} \varphi(x, y), \quad i, j=1, \ldots, k .
$$

The following theorem is proved in [13, Section 7.7]. 
Theorem A.1. Assume that at $\left(x_{0}, y_{0}\right) \in C$, the matrix $\left(\varphi_{i, j}\left(x_{0}, y_{0}\right)\right)$ is invertible. Then, there exists a neighborhood $U$ of $\left(x_{0}, y_{0}\right)$ such that if the support of a is a subset of $U$, one has

$$
I(\varphi, a)(x, \tau)=\left(\frac{2 \pi}{\tau}\right)^{k / 2} d(x) \mathrm{e}^{i \tau \varphi_{r}(x)} b(x, \tau)+O\left(\tau^{-\infty}\right) \quad \text { over } p(U),
$$

where $d, \varphi_{r}$ and $b(\cdot, \tau)$ are $C^{\infty}$ functions such that

- $d$ only depends on $\varphi$. In particular,

$$
d(x)=\operatorname{det}^{-1 / 2}\left[\frac{1}{i} \varphi_{j, k}(x, y)\right]_{j, k}, \quad \text { if }(x, y) \in C \cap U .
$$

- $\varphi_{r}$ is such that $\varphi(x, y) \equiv \varphi_{r}(x)$ on $U$ modulo a linear combination with $C^{\infty}$ coefficient of the functions $\partial_{y^{j}} \varphi$.

- $b(\cdot, \tau)$ has an asymptotic expansion for the $C^{\infty}$ topology of the form

$$
b_{0}(x)+\tau b_{1}(x)+\tau^{2} b_{2}(x)+\cdots .
$$

Furthermore, $b_{0}(x)=a(x, y)$ if $(x, y) \in C \cap U$.

In [13], the various terms of the asymptotic expansion are completely determined and not only their restriction at $p(C)$. But, in the applications, this leads to complicated computations that we prefer to avoid. Let us introduce an additional assumption.

Denote by $E_{(x, y)}$ the complexification of the tangent space to the fiber of $p$ at $(x, y)$. At $(x, y) \in C$, the tangent map to the section $d_{y} \varphi$ of $E^{*}$

$$
T_{(x, y)} d_{y} \varphi: T_{(x, y)} W \otimes \mathbb{C} \longrightarrow E_{(x, y)}^{*}
$$

is well defined. Assume that $\left(\varphi_{i, j}\right)$ is invertible along $C$, that is, the kernel $F_{(x, y)}$ of $T_{(x, y)} d_{y} \varphi$ satisfies

$$
\forall(x, y) \in C, \quad F_{(x, y)} \oplus E_{(x, y)}=T_{(x, y)} W \otimes \mathbb{C} .
$$

Assume furthermore that

$$
C \text { is a submanifold of } W \text { and } \quad T C \otimes \mathbb{C}=F \cap \bar{F} .
$$

Finally, these two assumptions imply that the restriction $p: C \rightarrow \mathbb{R}^{n}$ is an immersion. We assume it is an embedding.

Observe that when the phase takes real values, assumption (A.2) is a consequence of (A.1). We are interested in the opposite case, typically when the Hessian of the imaginary part of the phase is non-degenerate in the transverse directions to $C$, for instance, with

$$
\varphi(x, y)=x y+\frac{i}{2}\left(x^{2}+y^{2}\right) .
$$

We can also consider intermediary cases, for example, $\varphi(x, y)=x y+\frac{i}{2} y^{2}$.

Under the previous assumptions, when the amplitude $a$ vanishes to order $m$ along $C$, i.e., when the partial derivatives of $a$ of order $\leqslant m-1$ vanish 
along $C$, it follows from the result of $[\mathbf{1 3}]$ that the functions $b_{i}$ vanish to order $m-2 i$ along $C$. Furthermore, one can easily compute $b_{i}$ modulo a function vanishing to order $m-2 i+1$ along $C$.

To state the result, consider a free family $\partial_{1}, \ldots, \partial_{l}$ of complex tangent vectors to $W$ at $(x, y) \in C$ such that

$$
\operatorname{Vect}_{\mathbb{C}}\left(\partial_{1}, \ldots, \partial_{l}\right) \oplus(T C \otimes \mathbb{C})=F_{(x, y)} .
$$

If $a$ vanishes to order $m$ along $C$, we define the polynomial

$$
[a](Z, Y)=\sum_{|\alpha|+|\beta|=m} \frac{1}{\alpha ! \beta !}\left(\partial_{1}^{\alpha(1)} \cdots \partial_{l}^{\alpha(l)} \partial_{y^{1}}^{\beta(1)} \cdots \partial_{y^{k}}^{\beta(l)} a(x, y)\right) Z^{\alpha} Y^{\beta}
$$

at $(x, y) \in C$. Similarly, if $b(x)$ vanishes to order $l$ along $p(C)$, we set

$$
[b](Z)=\sum_{|\alpha|=l} \frac{1}{\alpha ! \beta !}\left(\left(p_{*} \partial_{1}\right)^{\alpha(1)} \cdots\left(p_{*} \partial_{l}\right)^{\alpha(l)} b(x)\right) Z^{\alpha}
$$

at $x \in p(C)$.

Theorem A.2. Under the assumptions (A.1) and (A.2), if a vanishes to order $m$ along $C$, then for every $i \leqslant \frac{m}{2}$, the function $b_{i}$ vanishes to order $m-2 i$ along $p(C)$. Furthermore,

$$
\left[b_{i}\right](Z)=\frac{1}{i !} \Delta^{i} A_{2 i}(Z, Y)
$$

at $(x, y) \in C$, where

- $[a](Z, Y)=\sum_{l=0}^{m} A_{l}(Z, Y)$ and $A_{l}$ is homogeneous of degree $l$ in $Y$ and of degree $m-l$ in $Z$.

- $\Delta=\frac{i}{2} \sum_{j, k} \varphi^{j, k}(x, y) \partial_{Y^{j}} \partial_{Y^{k}}$, with $\left(\varphi^{j, k}(x, y)\right)$ the inverse of $\left(\varphi_{j, k}(x, y)\right)$.

More intrinsically, denote by $\mathcal{I}^{m}(C) \subset C^{\infty}(W)$ the ideal of functions vanishing to order $l$ along $C$. Then, $\mathcal{I}^{m}(C) / \mathcal{I}^{m-1}(C)$ is isomorphic to the space of sections of the $m$-th symmetric power of the complex conormal bundle $\mathcal{N}^{*}(C)$. By (A.1), we have an isomorphism of vector bundle over $C$,

$$
\mathcal{N}^{*}(C) \simeq \mathcal{N}^{*}(p(C)) \oplus E^{*},
$$

which associates $\gamma$ and $\alpha \oplus \beta$ if

$$
\langle\gamma, U+V\rangle=\left\langle\alpha, p_{*} U\right\rangle+\langle\beta, V\rangle, \quad U \in F \text { and } V \in E .
$$

Consequently,

$$
\operatorname{Sym}_{m}\left(\mathcal{N}^{*}(C)\right)=\bigoplus_{l=0}^{m} \operatorname{Sym}_{m-l}\left(\mathcal{N}^{*}(p(C))\right) \otimes \operatorname{Sym}_{l}\left(E^{*}\right) .
$$

$\Delta=\frac{i}{2} \sum_{j, k} \varphi^{j, k} \partial_{y^{j}} \partial_{y^{k}}$ defines a section of $\operatorname{Sym}_{2}(E)$, so $\Delta^{i}$ acts as an operator

$$
\operatorname{Sym}_{2 i}\left(E^{*}\right) \longrightarrow \mathbb{C} \text {. }
$$


In Theorem A.2, we consider $[a]$ as a section of $\operatorname{Sym}_{m}\left(\mathcal{N}^{*}(C)\right)$ and $\left[b_{i}\right]$ as a section of $\operatorname{Sym}_{m-2 i}\left(\mathcal{N}^{*}(p(C))\right)$. Then we have

$$
\left[b_{i}\right]=\frac{1}{i !}\left(\operatorname{Id} \otimes \Delta^{i}\right) A_{2 i}
$$

where

$$
[a]=\sum_{l=0}^{m} A_{l}, \quad A_{l} \in C^{\infty}\left(C, \operatorname{Sym}_{m-l}\left(\mathcal{N}^{*}(p(C))\right) \otimes \operatorname{Sym}_{l}\left(E^{*}\right)\right) .
$$

\section{Acknowledgements}

We thank Y. Colin de Verdière who provided us his article $[\mathbf{9}]$ and suggested us to adapt his argument to the Toeplitz operators. This was actually one of our original motivations to develop the half-form formalism. We also thank F. Faure for his kind interest.

\section{References}

[1] F.A. Berezin, General concept of quantization, Comm. Math. Phys. 40 (1975), 153174.

[2] M. Bertelson, M. Cahen and S. Gutt, Equivalence of star products, Geometry and physics. Classical Quan. Grav. 14(1A) (1997), A93-A107.

[3] M. Bordemann, E. Meinrenken and M. Schlichenmaier, Toeplitz quantization of Kähler manifolds and $g l(N), N \rightarrow \infty$ limits, Comm. Math. Phys. 165(2) (1994), 281-296.

[4] D. Borthwick, T. Paul and A. Uribe, Semiclassical spectral estimates for Toeplitz operators, Ann. Inst. Fourier (Grenoble) 48(4) (1998), 1189-1229.

[5] L. Boutet de Monvel and V. Guillemin, The spectral theory of Toeplitz operators, Annals of Mathematics Studies, 99, Princeton University Press, Princeton, NJ, 1981.

[6] L. Charles, Berezin-Toeplitz operators, a semi-classical approach, Comm. Math. Phys. 239(1-2) (2003), 1-28.

[7] L. Charles, Quasimodes and Bohr-Sommerfeld conditions for the Toeplitz operators, Comm. Partial Diff. Eq. 28(9-10) (2003), 1527-1566.

[8] L. Charles, Semi-classical properties of geometric quantization with metaplectic correction, to appear in Comm. Math. Phys., http://www.institut.math.jussieu.fr/ ${ }^{\sim}$ charles/Articles/Half2.pdf, 2006.

[9] Y. Colin de Verdières, Bohr-sommerfeld rules to all orders, Ann. Henri Poincaré, 6(5) (2005), 925-936.

[10] J.J. Duistermaat, Oscillatory integrals, Lagrange immersions and unfolding of singularities, Comm. Pure Appl. Math. 27 (1974), 207-281.

[11] B. Fedosov, Deformation quantization and index theory, Mathematical Topics, 9, Akademie Verlag, Berlin, 1996.

[12] V. Guillemin, Star products on compact pre-quantizable symplectic manifolds, Lett. Math. Phys. 35(1) (1995), 85-89. 
[13] L. Hörmander, The analysis of linear partial differential operators. I, Grundlehren der Mathematischen Wissenschaften [Fundamental Principles of Mathematical Sciences], Distribution Theory and Fourier Analysis, 256, Springer-Verlag, Berlin, 2nd ed., 1990.

[14] A.V. Karabegov, Cohomological classification of deformation quantizations with separation of variables, Lett. Math. Phys. 43(4) (1998), 347-357.

[15] A.V. Karabegov and M. Schlichenmaier, Identification of Berezin-Toeplitz deformation quantization. J. Reine Angew. Math. 540 (2001), 49-76.

[16] B. Kostant, Quantization and unitary representations. I. Prequantization, in Lectures in Modern Analysis and Applications, III, 87-208, Lecture Notes in Math., 170, Springer, Berlin, 1970.

[17] D. McDuff and D. Salamon, Introduction to symplectic topology, Oxford Mathematical Monographs, The Clarendon Press Oxford University Press, New York, 2nd ed. 1998.

[18] R. Nest and B. Tsygan, Algebraic index theorem, Comm. Math. Phys. 172(2) (1995), 223-262.

[19] J.-M. Souriau, Structure des systèmes dynamiques, Maîtrises de mathématiques, Dunod, Paris, 1970.

[20] G.M. Tuynman, Quantization: towards a comparison between methods, J. Math. Phys. 28(12) (1987), 2829-2840.

[21] A. Weinstein, The Maslov gerbe, Lett. Math. Phys. 69 (2004), 3-9.

[22] S. Zelditch, Index and dynamics of quantized contact transformations, Ann. Inst. Fourier (Grenoble) 47(1) (1997), 305-363.

Université Pierre et Marie Curie-Paris6, UMR 7586 Institut de MathÉmatiques de Jussieu, PARIS, F-75005 FRANCE

E-mail address: charles@math.jussieu.fr

Received 16 February 2006 